\title{
Motor fluency shapes abstract concepts
}

\author{
Daniel Casasanto ${ }^{1,2,3}$ \& Evangelia G. Chrysikou ${ }^{4,5}$
}

${ }^{1}$ Max Planck Institute for Psycholinguistics, Nijmegen, $N L$

${ }^{2}$ Donders Institute for Brain, Cognition, \& Behaviour, Nijmegen, $N L$

${ }^{3}$ Department of Psychology, The New School for Social Research, New York, NY

${ }^{4}$ Psychology Department, University of Pennsylvania, Philadelphia, PA

${ }^{5}$ Center for Cognitive Neuroscience, University of Pennsylvania, Philadelphia, PA

People with different types of bodies tend to think differently in predictable ways, even about abstract ideas that seem far removed from bodily experience. ${ }^{1-4}$ Right- and lefthanders implicitly associate positive ideas like goodness and honesty more strongly with their dominant side of space, the side on which they can interact with their environment more fluently, and negative ideas with their non-dominant side. ${ }^{1,2}$ This suggests a role for motor experience in shaping abstract thoughts. Yet, previous evidence is also consistent with an experience-independent account. Here we show that right-handers' tendency to associate 'good' with right and 'bad' with left can be reversed due to both long- and shortterm changes in motor fluency. Among stroke patients who were right-handed prior to unilateral cerebrovascular accident (CVA), those with left-hemiparesis (following right CVA) associated good with right, but those with right-hemiparesis (following left CVA) associated good with left, like natural left-handers. A similar pattern was found in healthy right-handers whose right or left hand was temporarily handicapped in a laboratory training task. Motor experience influences judgments of good and bad, overriding any predispositions due to natural handedness. Even highly abstract ideas depend, in part, on how people interact with the physical world.

When people talk about good and bad things they often use spatial metaphors. Across many languages and cultures, ideas with positive and negative emotional valence can be expressed in terms of up and down (on top of the world means 'happy,' down in the dumps 
means 'sad') or right and left (the right answer means 'correct,' two left feet means 'clumsy')., Beyond using spatial language, people also think about good and bad things using mental representations of space and motion, as evidenced by behavioural experiments. ${ }^{6-11}$ People express more pride in their accomplishments when they are induced to sit up tall as opposed to slumping down. ${ }^{6}$ Likewise, they retrieve more positive emotional memories when asked to move objects upward and more negative memories when asked to move them downward. ${ }^{7}$ Implicitly, people conceptualize good things as higher and bad things as lower on an imaginary spatial continuum, consistent with patterns in language.

People also spatialize good and bad on the horizontal axis, but not always as expressions in language suggest. Rather, associations of abstract qualities like goodness and honesty with left-right space vary according to people’s handedness. When asked to decide which of two products to buy, which of two job applicants to hire, or which of two alien creatures looks more trustworthy, right-handers tend to prefer the product, person, or creature presented on their right side but left-handers tend to prefer the one on their left. ${ }^{1}$ This pattern persists even when people make judgments orally, without using their hands to respond.

Links between valence and left-right space can also be observed beyond the laboratory, in spontaneous speech and gesture. In the final debates of the 2004 and 2008 US presidential elections, which involved two right-handers (Kerry, Bush) and two left-handers (Obama, McCain), right-handed candidates produced a higher proportion of right-hand gestures during positive-valence speech and left-hand gestures during negative-valence speech; the opposite pattern was found in the left-handed candidates. ${ }^{2}$

Overall, these patterns cannot be predicted or explained based on conventions in language and culture, which consistently associate good with right and bad with left. ${ }^{1,12}$ Rather, 
results support the body-specificity hypothesis, showing that people with different kinds of bodies think differently in predictable ways, even about highly abstract ideas. ${ }^{1-4}$

Yet, an important question remains: Why do right- and left-handers associate good and bad things with opposite sides of space? On one possibility, people’s experience of interacting with the physical environment more fluently on their dominant side and less fluently on their non-dominant side could lead to the formation of implicit associations in memory. More fluent perceptuo-motor processing often leads to more positive feelings and evaluations. ${ }^{13-16}$ The dominant side may be good in people’s minds because dominant-side actions tend to be more fluent than non-dominant side actions. On an alternative possibility, different spatial mappings of good and bad could result from genetically-determined neurological differences between right- and left-handers: That which gives rise to handedness could also give rise to handednessrelated differences in judgments. ${ }^{1,17-22}$ In principle, the body-specific association between space and valence could be experience-independent.

Here we investigated a role for motor experience in establishing implicit associations between space and valence. We tested whether long- and short-term changes in motor fluency can influence judgments about the spatial correlates of good and bad. To evaluate the effects of long-term changes, we tested stroke patients with hemiparesis (weakness or paralysis on one side of the body) following unilateral CVA. All patients $(n=10)$ were right-handed prior to brain injury. Right-hemisphere CVA led to left hemiparesis in four of the patients, preserving their natural right-handedness. Left-hemisphere CVA led to right hemiparesis in the other six patients making them effectively left-handed, post-stroke. Amid filler tasks, patients performed a two-question diagram task known to elicit contrasting space-valence judgments from healthy right- and left-handers. ${ }^{1}$ Patients saw a cartoon character's head in the center of a page between two empty boxes, one on his right and the other on his left (Supplementary Fig. 1). They were 
told that the character loves zebras and thinks they are good, but hates pandas and thinks they are bad (or vice versa). Participants indicated where the character would put each of the animals if he were going to put the good animal in one box and the bad animal in the other. The assignment of positive/negative valence to the panda and zebra was counterbalanced across participants, as was the order in which participants were asked about the good and bad animals. They responded orally, not manually; there was no manual motor component to the diagram task, eliminating any trivial online effects of motor fluency on responses.

For 9 out of the 10 participants, judgments varied according to their post-stroke motor fluency (sign test on 9 vs. $1, P=.02$ ). All four left-hemiparesis patients (100\%) indicated that the good animal should go in the right box and the bad animal in the left box, consistent with their pre- and postmorbid right-hand dominance. By contrast, five of the six right-hemiparesis patients (83\%) indicated that the good animal should go in the left box and the bad animal in the right box, contrary to their premorbid right-handedness but consistent with their post-stroke lefthand dominance (association of post-stroke dominant hand [right, left] with response [good=right, bad=right]: Fisher's exact test $P=.05,2$-tailed; Pearson's $\chi^{2}=8.67, \mathrm{df}=1, \mathrm{P}=$ $.003)$.

Prolonged reversal of natural hand dominance can reverse right handers' usual tendency to associate good with right. Yet, the stroke patients' brain injuries not only led to lasting changes in motor fluency but also to long-term neural reorganization. The locus of CVAs varied widely across patients (see Table 1); therefore it would be complicated to explain the present results based on neural changes, per se, as opposed to resultant changes in motor fluency. Still, we cannot rule out the possibility of such an explanation, at least in principle.

In order to determine whether changes in motor fluency can affect space-valence mappings independent of any long-term neural reorganization, we conducted a two-part training 
experiment. Healthy right-handed participants $(n=55)$ first performed a motor fluency task (training phase), and then the same diagram task used with the stroke patients (test phase).

For the training phase, participants arranged dominoes in a symmetrical pattern on a tabletop. They placed dominoes on horizontal rows of dots, moving both hands symmetrically. They were instructed to fill as many rows of dots as possible in 12 minutes, as a test of motor coordination.

Participants were randomly assigned to wear a bulky ski glove on either their right or left hand, with the other ski glove dangling from the wrist of the gloved hand (see Supplementary Fig. 2). This made grasping and positioning dominoes much more difficult with one hand than the other. For about half of the participants, wearing the glove on their left hand preserved their natural right-handedness. For the other half of the participants, wearing the glove on their right hand turned them transiently 'left-handed,' reversing the normal relationship between motor fluency and their left and right sides of space.

After completing the domino task, participants removed the ski glove. They were led into a different room, by a different experimenter, for a series of three unrelated questionnaire studies, one of which was the 'animals' task. Like the stroke patients, the ski glove-trained participants indicated where the cartoon character would place the good and bad animals orally, without using their hands to respond, eliminating trivial forms of transfer from the training phase to the test phase.

Motor experience influenced participants’ spatial mapping of good and bad. Participants whose left hand was handicapped during training (preserving their natural right-handedness) tended to assign the good animal to the right box, whereas participants whose right hand was handicapped (making them effectively left-handed) tended to assign the good animal to the left box $\left(\right.$ Wald $\chi^{2}=8.01, \mathrm{df}=1, \mathrm{P}=.005, \mathrm{OR}=5.67,95 \%$ C.I. $=1.71-18.83$; Fig. 1 ). Extensive 
debriefing ensured that this effect was not due to participants detecting any connection between the training and test phases, which were ostensibly unrelated.

Results of the ski glove study demonstrate a causal relationship between motor fluency and judgments about the abstract ideas of goodness and badness. The effects of short-term motor training are presumably temporary, but the same associative learning mechanisms that partly determine people's performance in the laboratory may result in the long-term changes we found in stroke patients, and may shape natural right- and left-handers' space-valence mappings in the course of ordinary motor experience. Using our lopsided bodies, and therefore interacting with the physical environment more fluently on one side of space than the other, may serve as a kind of natural 'motor training.'

This does not rule out the possibility that innate neurobiological factors could also contribute to the body-specific mappings observed in natural righties and lefties. But the fact that right-handers' judgments reversed after long- or short-term training demonstrates that motor experience is sufficient to determine the direction of space-valence associations, and even to temporarily overwhelm any innate predisposition to associate 'good' with one's naturally dominant side.

Motor fluency has been linked previously with preferences for things that people can act on with their hands. People prefer graspable objects like spatulas when their handles are oriented so as to make them easy to grasp. ${ }^{5}$ Skilled typists prefer pairs of letters that are easy to type, even when they are not typing. ${ }^{13}$ These effects can be readily explained in terms of motor affordances: People mentally simulate performing the action that an object would afford if they were to act on it, such as picking up a spatula or typing letters, and their preference judgments vary according to how fluent this action would be. 
Yet, motor tendencies also predict judgments about abstract ideas and things people can never manipulate with their hands, as when left- and right-handers attribute more intelligence or honesty to alien creatures depicted on their dominant side of a page. ${ }^{1}$ In the present study, changes in motor fluency influenced participants' judgments about intangible qualities (goodness and badness) of imaginary zoo animals (zebras and pandas, which would be unlikely to afford manipulation with one hand even if they were real). These results demonstrate a causal link between manual motor fluency and preference judgments, but suggest that this link is not necessarily mediated by mental simulation of action affordances. Associations between emotional valence and left-right space may be established through habits of fluent and disfluent hand actions, but these associations generalize to influence judgments of things people can never see or touch. It remains a challenge for ongoing research to characterize the neurocognitive mechanisms by which physical experience generalizes to shape abstract conceptions of good and bad. 


\section{References}

1. Casasanto, D. Embodiment of abstract concepts: good and bad in right- and left-handers. $J$ Exp. Psychol. Gen., 138, 351-367 (2009).

2. Casasanto, D. \& Jasmin, K. Good and bad in the hands of politicians: spontaneous gestures during positive and negative speech. PLoS ONE, 5, e11805 (2010).

3. Willems, R. M., Hagoort, P. \& Casasanto, D. Body-specific representations of action verbs: neural evidence from right- and left-handers. Psychol. Sci., 21, 67-74 (2010).

4. Willems, R. M., Toni, I., Hagoort, P. \& Casasanto, D. Body-specific motor imagery of hand actions: neural evidence from right-and left-handers. Front. Hum. Neurosci., 3, 1-9 (2009).

5. Lakoff, G. \& Johnson, M. Philosophy in the Flesh: The Embodied Mind and Its Challenge to Western Thought (Chicago: University of Chicago Press, 1999).

6. Stepper, S. \& Strack, F. Proprioceptive determinants of emotional and nonemotional feelings. J. Pers. Soc. Psychol., 64, 211-220 (1993).

7. Casasanto, D. \& Dijkstra, K. Motor action and emotional memory. Cognition, 115, 179-185 (2010).

8. Riskind, J. H. \& Gotay, C. C. Physical posture: could it have regulatory or feedback effects on motivation and emotion? Motiv. Emotion, 6, 273-298 (1982).

9. Meier, B. P. \& Robinson, M. D. Why the sunny side is up: associations between affect and vertical position. Psychol. Sci., 15, 243-247 (2004).

10. Tracy, J. L. \& Matsumoto, D. The spontaneous expression of pride and shame: evidence for biologically innate nonverbal displays. Proc. Natl Acad. Sci. USA, 105, 11655-11660 (2008).

11. Crawford, L. E. Conceptual metaphors of affect. Emotion Rev., 1, 129-139 (2009).

12. Clark, H. H. in Cognitive Development and the Acquisition of Language (ed. Moore, T. E.) 27-63 (New York: Academic Press, 1973). 
13. Reber, R., Winkielman, P. \& Schwarz, N. Effects of perceptual fluency on affective judgments. Psychol. Sci., 9, 45-48 (1998).

14. Beilock, S. L. \& Holt, L. E. Embodied preference judgments: can likeability be driven by the motor system? Psychol. Sci., 18, 51-57 (2007).

15. Oppenheimer, D. M. The secret life of fluency. Trends Cogn. Sci., 12, 237-241 (2008).

16. Ping, R. M., Dhillon, S. \& Beilock, S. L. Reach for what you like: the body's role in shaping preferences. Emotion Rev., 1, 140-150 (2009).

17. Kinsboume, M. in Asymmetrical Function of The Brain (ed. Kinsbourne, M.) 553-556 (New York: Cambridge University Press, 1978).

18. Kinsbourne, M. \& Bemporad, B. in The Psychobiology of Affective Development (eds. Fox, N. A. \& Davidson, R. J.) 259-291 (Hillsdale, N J: Erlbaum, 1984).

19. Davidson, R. J. \& Fox, N. A. Asymmetrical brain activity discriminates between positive and negative affective stimuli in human infants. Science, 218, 1235-1237 (1982).

20. Gesell, A. \& Ames, L. B. The development of handedness. Pedagogical Seminary and J. Genet. Psychol., 70, 155-175 (1947).

21. Coren, S. The Left-Hander Syndrome: The Causes and Consequences of Left Handedness (New York: Random House, 1992).

22. Corballis, M. \& Beale, I. Psychology of Left and Right (Hillsdale, NJ: Erlbaum, 1976).

23. Oldfield, R. C. The assessment and analysis of handedness: the Edinburgh inventory. Neuropsychologia, 9, 97-113 (1971).

24. Wilson, T. D. \& Nisbett, R. E. The accuracy of verbal reports about the effects of stimuli on evaluations and behavior. Soc. Psychol., 41, 118 -131 (1978). 
25. Van Strien, J. W. Classificatie van links- en rechtshandige proefpersonen / Classification of left-handed and right-handed test subjects. Nederlands Tijdschrift voor de Psychologie en haar Grensgebieden, 47, 88-92 (1992).

Supplementary Information accompanies the paper on www.nature.com/nature.

Acknowledgments This research was supported in part by an NRSA fellowship (\#F32MH072502) to D.C., a grant from the Consejería de Innovación, Ciencia y Empresa, Junta de Andalucía and the European Regional Development Fund (P09-SEJ-4772) to D.C., and by an NIH grant (\#R01MH70850) to Sharon L. Thompson-Schill.

Author Contributions The study was initiated by D. C. and E. G. C. Data were compiled by D. C. and E. G. C.; D.C. conducted the analyses and drafted the manuscript. Both authors discussed the results and contributed to the writing of the manuscript.

Author Information Reprints and permissions information is available at www.nature.com/reprints. The authors declare no competing financial interests. Correspondence and requests for materials should be addressed to D.C. (casasanto@alum.mit.edu). 


\section{Figure legend}

Figure 1. Effects of short-term motor training on the spatial correlates of good and bad. Results of the dominoes-ski glove experiment showed strong effect of motor training, by binary logistic regression (Wald $\chi^{2}=8.01, \mathrm{df}=1, p=.005$ ). Right-handed participants who wore the cumbersome glove on their left hand during motor training were more likely to assign the good animal to the right box, like natural right-handers $(z=2.29, p=.01$; left columns). Right-handers who wore the glove on their right hand were more likely to assign the good animal to the left box, like natural left-handers ( $z=1.84, p=.03$; right columns). 
Table 1.

\section{Table 1. Patient Demographic and Clinical Information for Experiment 1}

\begin{tabular}{|c|c|c|c|c|c|}
\hline Patient ID & Gender & $\begin{array}{c}\text { Age } \\
\text { (years) }\end{array}$ & $\begin{array}{c}\text { Education } \\
\text { (years) }\end{array}$ & $\begin{array}{c}\text { Time post-stroke } \\
\text { (years) }\end{array}$ & Locus of unilateral CVA \\
\hline TS474 & $\bar{F}$ & 50 & 11 & 8 & R parietal cortex \\
\hline KG593 & $\mathrm{F}$ & 48 & 12 & 4 & $\begin{array}{l}\mathrm{R} \text { frontal, temporal, parietal } \\
\text { cortex, and basal ganglia }\end{array}$ \\
\hline $\mathrm{NH} 192$ & $\mathrm{~F}$ & 71 & 12 & 12 & $\mathrm{R}$ thalamus \\
\hline TN694 & $F$ & 31 & 12 & 7 & $\begin{array}{l}\mathrm{R} \text { frontal, parietal cortex, and } \\
\text { basal ganglia }\end{array}$ \\
\hline QN435 & M & 55 & 12 & 8 & $\begin{array}{l}L \text { frontal, parietal cortex, and } \\
\text { pons }\end{array}$ \\
\hline XK342 & $\mathrm{F}$ & 56 & 12 & 10 & $\begin{array}{l}\text { L frontal, parietal white matter; } \\
\text { L occipital cortex and centrum } \\
\text { semiovale }\end{array}$ \\
\hline CD141 & $\mathrm{F}$ & 51 & 16 & 11 & $\begin{array}{l}\mathrm{L} \text { insular, perisylvian cortex, } \\
\text { and basal ganglia }\end{array}$ \\
\hline TD360 & M & 56 & 12 & 9 & $\begin{array}{l}\text { L temporal cortex, centrum } \\
\text { semiovale, and basal ganglia }\end{array}$ \\
\hline LM292 & M & 63 & 18 & 10 & L basal ganglia \\
\hline BC236 & M & 63 & 18 & 17 & $\begin{array}{l}\mathrm{L} \text { frontal, parietal cortex and } \\
\text { basal ganglia }\end{array}$ \\
\hline
\end{tabular}

Notes: $F=$ Female,$M=$ Male,$R=$ right,$L=$ left, $C V A=$ cerebrovascular accident. The location of the CVA was determined by the attending neurologist at the Hospital of the University of Pennsylvania, following visual inspection of post-stroke clinical computerized axial tomography (CAT) or magnetic resonance imaging (MRI) scans. 


\section{Methods}

\section{Experiment 1.}

Participants. Ten stroke patients ( 4 men, 6 women; mean age $=54.4$ years), all native English speakers, participated in this study after providing informed consent. All patients were strongly right-handed prior to stroke, according to their original post-stroke clinical assessment with the Edinburgh Handedness Inventory (pre-stroke EHI scores for all patients $\geq 90$ ). ${ }^{22}$ Postmorbidly, the 4 patients who had suffered right unilateral CVAs (left-hemiparesis) remained right-handed (mean post-stroke EHI $=87.50 \pm 2.5$ ), whereas the 6 patients who had suffered left unilateral CVAs (right-hemiparesis) essentially became left-handed (mean post-stroke EHI= -76.67 $\pm 10.54)$. EHI scores differed between the two groups $(t(8)=12.32$, P = .001; Mann-Whitney U test, $Z=-2.60, \mathrm{P}=.01)$. Basic demographic and lesion location information by patient is presented in Table 1.

Materials and procedure. The patients were tested at the University of Pennsylvania Hospital. They responded orally to a two-question diagram task, shown previously to elicit a bodyspecific pattern of responses in healthy left- and right-handers. Materials and procedures were identical to those used in Experiment 3 of Casasanto (2009), except that the original Dutch instructions were translated into English (Supplementary Fig. 1). ${ }^{1}$

Debriefing. After testing, the patients were asked why they assigned the good animal to the box that they chose. No one mentioned hand dominance or motor fluency. They were then asked explicitly whether their handedness or the way they use their hands could have influenced their decisions. The most common answer $(n=4)$ was “I don’t know.” Three participants indicated that it was possible the way they use their hands could have influenced their judgments; importantly, they only agreed to this possibility after it was explicitly suggested to them, and even then they only responded "maybe.” The remaining three patients expressly 
denied that handedness or motor habits could have influenced their judgments, even though all three responded in accordance with the experimental hypothesis.

In summary, no patient reported having responded on the basis of their pre-stroke handedness or their post-stroke motor fluency, even when this possibility was suggested to them explicitly. This is consistent with previous research showing that people are largely unaware of the spatio-motor factors that influence their preference judgments. ${ }^{1,24}$

\section{Experiment 2.}

Participants. Fifty-five native Dutch speakers from the Radboud University community participated in exchange for payment. Participants were recruited in two cohorts (Cohort 1: $n=$ 28; Cohort 2: $n=27$ ). Data from Cohorts 1 and 2 were initially analyzed separately, to provide an internal replication of this study. Since no difference in the strength of the training effect was found between cohorts, their data were combined for subsequent analyses.

All participants were right-handed according to pre-screening and to a Dutch adaptation of the EHI, administered at the end of the testing session. ${ }^{22,25}$ Two of the participants were excluded from analysis on the basis of the debriefing (see below). For the remaining 53 participants, the mean EHI score was $78.73 \pm 2.59$. Twenty-six of the participants (EHI=82.20 \pm 3.25 ) were randomly assigned to wear the left ski glove during training, and twenty-seven $(\mathrm{EHI}=75.39 \pm 3.99)$ to wear the right glove. EHI scores did not differ significantly between groups $(t(51)=1.32, n s)$.

\section{Materials and procedure}

Training phase. Participants were seated at a table, the top of which was covered with a mat, $120 \mathrm{~cm}$ wide $\times 60 \mathrm{~cm}$ deep, with 80 dots spaced approximately $12 \mathrm{~cm}$ apart, in 8 horizontal rows. In the center of the mat, on the edge closest to the participant, there was a cardboard box containing two sets of dominoes (84 pieces). Ostensibly as a test of 'motor coordination,' 
participants were instructed to place dominoes on dots as quickly as possible for 12 minutes, under the following conditions.

Each participant was assigned to wear a bulky ski glove on either the right or left hand, with the other ski glove dangling from the same wrist. To avoid calling attention to the assignment of the glove to one hand or the other, the experimenter handed the participant only one of the gloves, and then fastened the second glove to the wrist of the first once the participant had put it on. Participants removed the dominoes from the box in pairs, one in each hand, and were instructed to place them on the dots in a symmetrical pattern, moving the hands in synchrony, using the left hand to place dominoes on the left side of the table and the right hand on the right side of the table. Dominoes were placed on dots standing upright, with the spots facing the participant. If dominoes were knocked over, the participant had to repair the arrangement before proceeding, using the appropriate hand. They were not allowed to use the free hand to prevent the dangling ski glove from knocking over the dominoes, or otherwise to help the gloved hand. Participants knew that they were videotaped. Although some participants occasionally cheated, using the free hand to help position dominoes between the fingers of the gloved hand, in general all participants complied closely with these instructions.

Test phase. After the training phase, participants were escorted to a different room, where they were tested by a different experimenter on three brief, unrelated questionnaire studies. The change of location and personnel was intended to enhance the impression that the training and test phases were unrelated. For the relevant questionnaire, participants saw the same diagram used in Experiment 1, and responded to the same prompts (in Dutch rather than English). 
Debriefing. Before participants were dismissed, they were debriefed extensively by the second experimenter about the purpose of the experiments, and also asked standard demographic questions as fillers. Even though the participants were asked explicitly whether they noticed any relationships among the experiments, only 2 of the 55 participants reported suspecting any connection between the critical training and test phases. Data from these participants were excluded from further analyses. According to the debriefing data, the 53 participants included in the analyses were unaware of any connection between the training and test phases, and were unaware their responses on the good-bad animals task were influenced by their previous motor experience. 


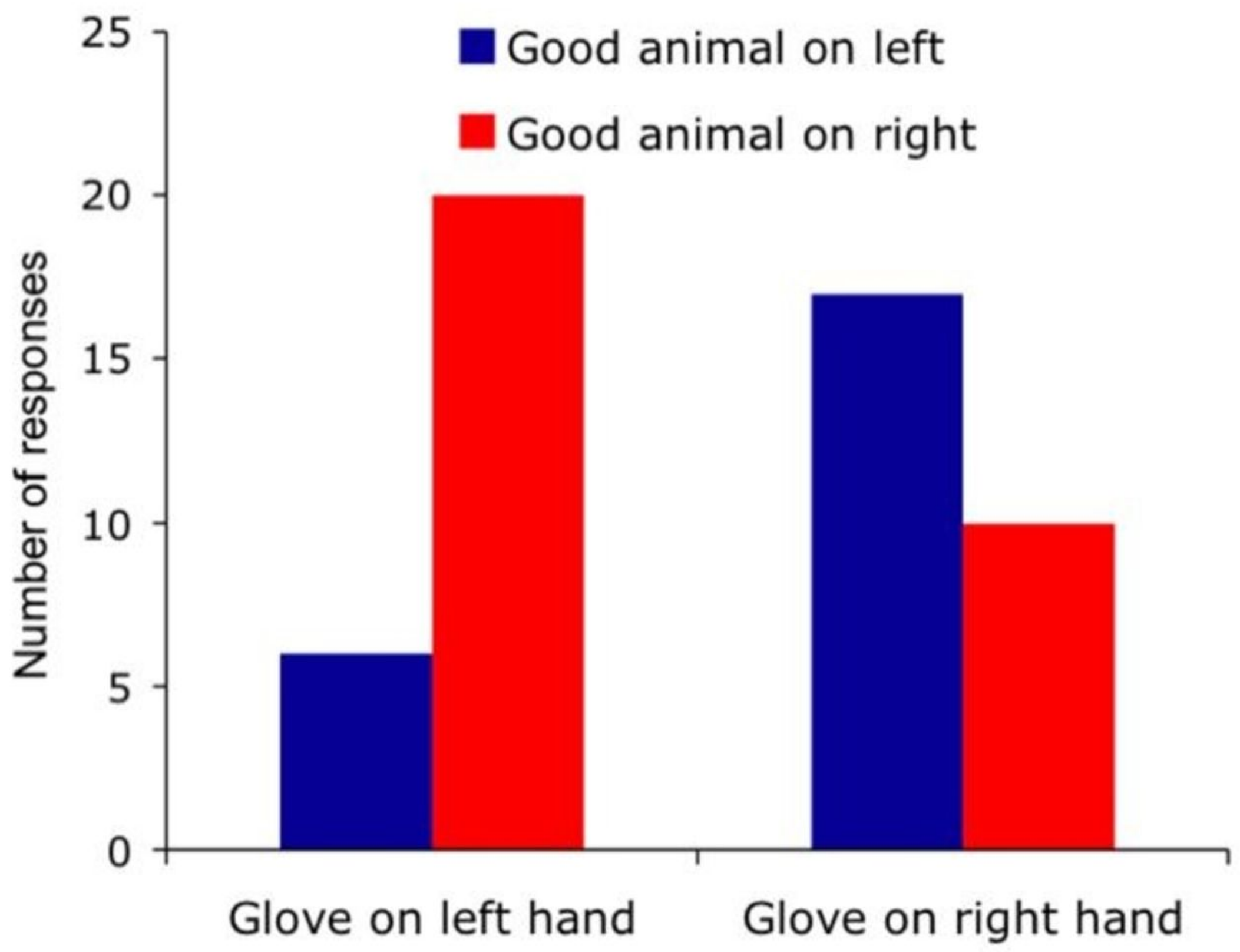

